\title{
HYALURONIC ACID FILLER INJECTION FOR COSMETIC PURPOSE: SKIN NECROSIS COMPLICATION AND TREATMENT - CASES REPORTS
}

\author{
Vu Ngoc Lam*, Nguyen Quang Duc*, Le Diep Linh*, Le Thi Thu Hai*
}

\section{ABSTRACT}

Background: Hyaluronic acid (HA) fillers for aesthetic are generally considered safe and the use of dermal fillers for soft tissue augmentation has become a very popular technique in aesthetic practices. Dermal fillers temporarily remove the appearance of rhytids and reduce the depth of skin folds. This is one of the most effective method for facial improvement used in Viet Nam nowadays. However, complications such as skin necrosis, blind... may happen and hence any filler injection practitioner need to be aware of such side effects, contraindications and precaution to be adopted while using fillers. Even with the most experienced of injectors, adverse effects can and do occur ranging from mild bruising to severe injection necrosis. Aims: HA filler injectors should be able to prevent and treat the severe complication of skin necrosis and detect impending necrosis after injection of a augmentation filler. Materials and Methods: Cases report of 3 patients who were HA filler injection for nose augmentation and had suffered from skin necrosis. These patients were followed for 3-6 months from time of injection of hyaluronic acid filler to complete healing of wound. Results: Complete wound healing were achieved with early recognition and institution of treatment, surgical procedures applied in some severe cases. The functional of the noses are remained but most of the patients have bad scar on the nose. Discussion: We review cases report of injection necrosis and methods used to prevent and treat this complication. Conclusion: Early recognition of vascular necrosis with specific

* 108 Military Central Hospital

Responsible person: Vu Ngoc Lam

Email: vungoclamb8@gmail.com

Date of receipt: 03/6/2021

Date of scientific judgment: 05/7/2021

Reviewed date: $17 / 8 / 2021$ protocol for treatment after injection necrosis with hyaluronic acid fillers improves the outcome of wound healing.

Keywords: HA fillers, side effects

\section{INTRODUCTION}

In Viet Nam as well as all over the world, injection of HA fillers is one of the most popular performed procedures in aesthetic specialty. According to data published by the American Society of Plastic Surgeons (ASPS) in 2014, soft tissue filler injections increased by $253 \%$ since the year 2000 with $3 \%$ increase from the year 2013 (2.3 million) [1]. Hyaluronic acid (HA) fillers constituted $78.3 \%$ of all injectable dermal fillers with $7.5 \%$ increase from the previous year. HA forms an integral part of the natural extracellular matrix which is found in high amounts in several connective tissues including the skin, the vitreous humor of the eye and the synovial fluid. Chemically, HA is a linear polysaccharide composed of repeating disaccharide units of glucuronic acid and $\mathrm{N}$ - acetylglucosamine. $\mathrm{HA}$ is considered to be the most popular dermal filler to replace volume loss due to normal aging for several reasons including: its hygroscopic property, biocompatibility and reversibility. Over the past several decades, various forms of HA fillers have been developed and they differ in many aspects including: the type and degree of crosslinking, gel viscosity, gel hardness, gel consistency, extrusion force, total HA 


\section{VIETNAM MEDICAL JOURNAL №1\&2/2021}

concentration and duration of presence in the skin [3]

As the usage of dermal fillers is expanding, complications will likely increase. Even in the hands of an experienced injector, various complications can occur. Fortunately, most of the complications associated with HA fillers are mild, transient and reversible. Injection technique related adverse effects are the most commonly seen. Maximizing injection technique and thorough understanding of potential complications and their management can help avoid, identify and manage them when they do occur. We would like to introduce the treatment of 3 patients with filler injection complication in 108 Military Central Hospital (108 MCH), Hanoi, Vietnam.
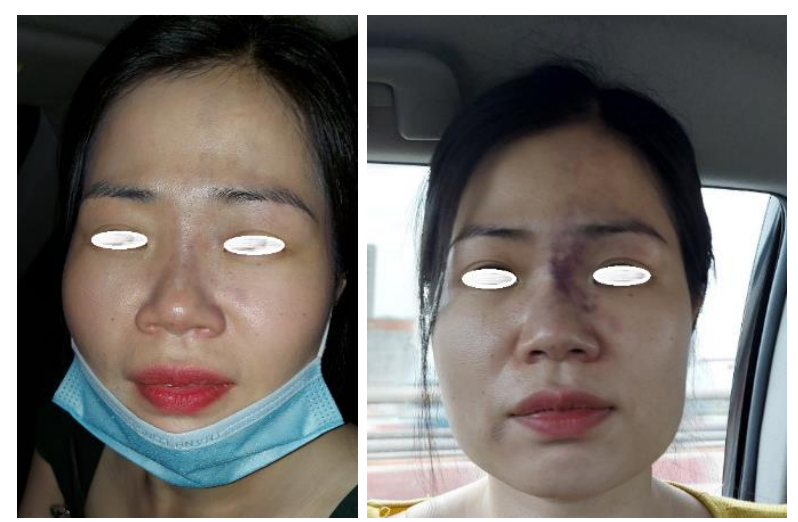

Pictures 1-2. Left: After 30 minutes of injection; Right: 4 hours later

- Check in symptom: The bruise lesion along her nose and came to the medial corner of upper eye lid, eye brow and at the low middle forehead.

Eye examination result: no vision loss.

- Treatment at $108 \mathrm{MCH}$ : She was treated by following protocol:

1. Quinolone antibiotic: Ciprofloxacin $500 \mathrm{mg} \mathrm{x}$ twice a day (7 days)

2. Solumedrol IV $40 \mathrm{mg} x$ once a day (3 days)

\section{CLINICAL CASES}

\subsection{Case 1. A 37-year-old lady}

- History: She was injected 1cc HA filler on the nose by a none medical injector at a spa for the purpose of nose augmentation. About 30minutes after injection she felt numbness, painful and swollen at the left upper eye lid, the sight was normal. 4 hours later, the skin on the nose changed the color to red. The area of red color increase quickly and became bruised. She was injected 100UI of Hyaluronidase for all the nose area and antibiotic 12 hours after HA injection and was taken to 108 Military Central Hospital for the treatment 24 hour later.

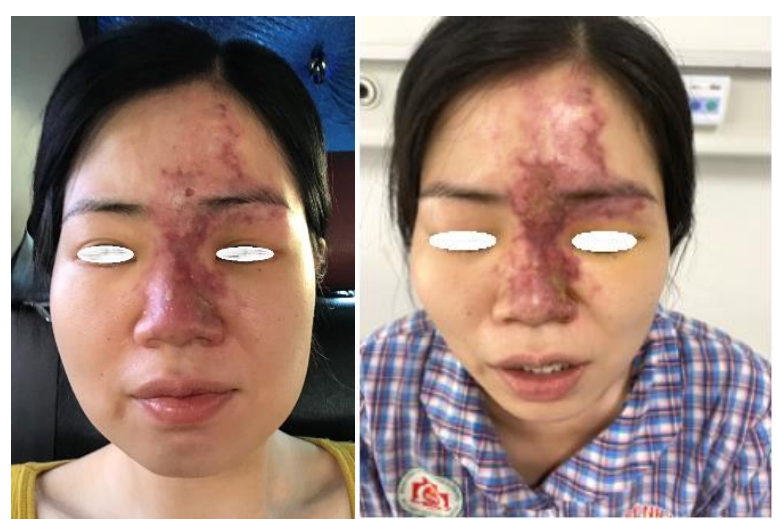

Pictures 3-4. Left: After Hyaluronidase injection; Right: At check in time

3. Praxilen $200 \mathrm{mg} \mathrm{x}$ twice a day (7 days)

4. Laser He-Ne $1 \mathrm{j} / 24$ hours (3 days)

5. Wet sponge with $\mathrm{NaCl} 0,9 \%$ on the lesion 8-10 hours a day (7 days)

6. No more Hyaluronidase injection.

- Results: After 2 day the lesion seem be smaller, the bruise reduced, patient felt much better. There was a small epithelial necrosis on the alar. 


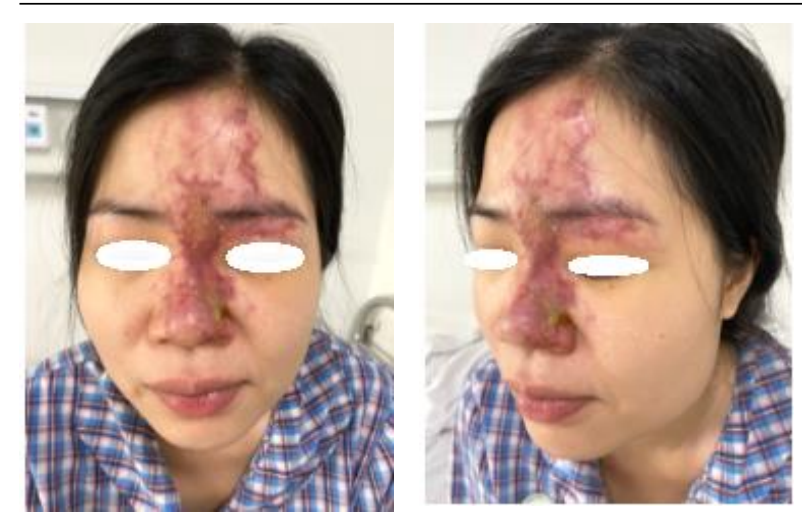

Picture 5-6: 2 days after treatment

- In 5 following days, the necrosis area narrowed and no surgical procedure need to apply. The epithelialization completed after 7 days of the treatment.
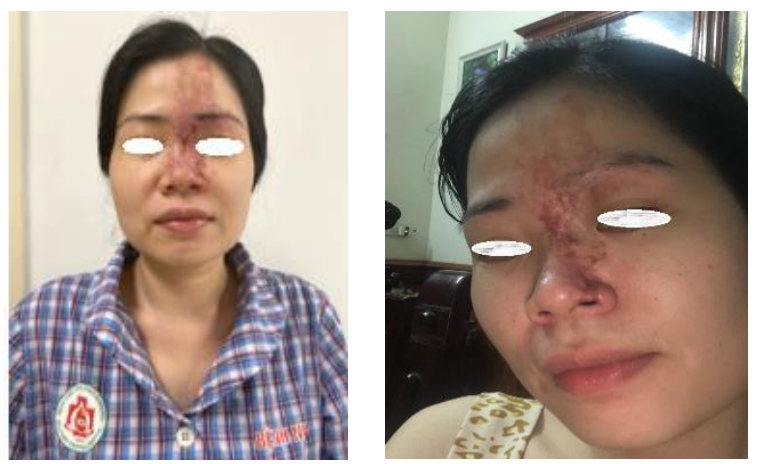

Picture 7 -8: After 7 days and 3 months

2.2. Case 2. A 33-year-old lady

- History: Injected 2cc filler (non-identify brand name of the filler) on the nose by a none medical injector at a spa for the same purpose of nose augmentation. Right after injection she felt hurt and swollen at the left upper eye lid, the sight was normal. 6 hours later, the skin on the nose changed the color to red. The area of red color expanded quickly and became bruised and the red color appeared in the medial sclera of left eye.

She was treated herself with antibiotic (not prescript by any doctors) for 5 days. The lesion came much worse and then she hospitalized to $108 \mathrm{MCH}$ for treatment.

- Check in symptom: The lesion occupied almost of her nose and came to the medial corner of upper eye lid, eye brow and at the low middle forehead. Most of the skin on the lesion was necrosis in different levels.

Eye examination result: The medial sclera of the left eye bruised but no vision loss.
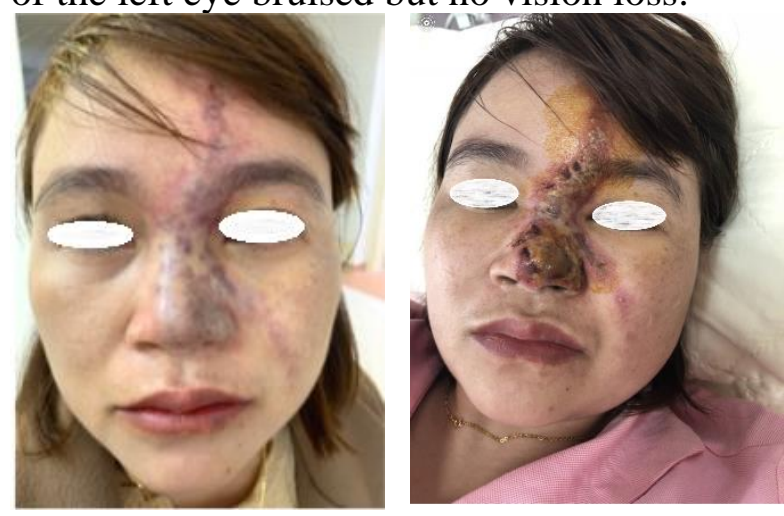

Picture 9-10: Lesion at 6 hours after filler injection and at check in time

- Treatment at $108 \mathrm{MCH}$ : She was treated by following protocol:

1. Quinolone antibiotic: Ciprofloxacin $500 \mathrm{mg} \mathrm{x}$ twice a day (7 days)

2. Solumedrol IV $40 \mathrm{mg} x$ once a day (3 days)

3. Praxilen $200 \mathrm{mg} \mathrm{x}$ twice a day (7 days)

5. Wet sponge with $\mathrm{NaCl} 0,9 \%$ on the lesion 8-10 hours a day

Surgical intervention: Necrosis tissue was debrided everyday in 2 weeks until the epithelialization almost completed.

She was discharged to continue to look after the lesion with skin care products (Scar gel) by herself.
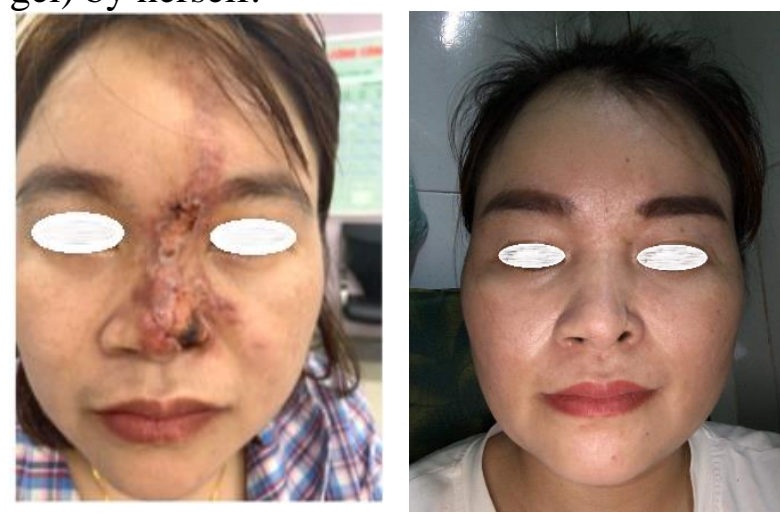

Picture 11-12: Day 10th of the treatment and 1 year later 
2.2. Case 3. A 23 year old girl.

- History: Injected 1cc filler (non-identify brand name of the filler) on the nose by a none medical injector at a spa for nose augmentation. After 60 minutes of injection, signs of pain appeared at the base of the nose, redness of the nose area spread to both cheeks and forehead. Normal vision.

The patient was brought to $108 \mathrm{MCH} 6$ hours later and injected with 100 UI Hyaluronidase into the nose, forehead, and cheeks. After that, the following treatment regimen is used:

1. Quinolone antibiotic: Ciprofloxacin $500 \mathrm{mg} \mathrm{x}$ twice a day (7 days)

2. Solumedrol IV $40 \mathrm{mg} x$ once a day (3 days)

3. Praxilen $200 \mathrm{mg} \mathrm{x}$ twice a day (7 days)

4. Laser He-ne $1 \mathrm{j} / 24$ hours (3 days)

5. Wet sponge with $\mathrm{NaCl} 0,9 \%$ on the lesion 8-10 hours a day (7 days)

The lesion gradually subsided with signs of reduction in the area of the bruised area, without skin necrosis.

The patient was discharged after 1 week of treatment.
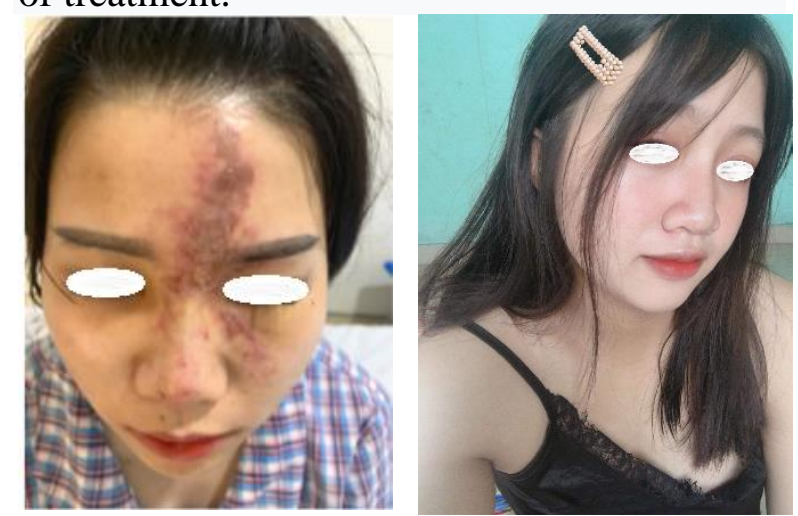

Picture 13-14: Lesion at 6 hours after filler injection and 2 years later

\section{DISCUSSION}

- Filler injection complications can be divided into early and delayed onset complications according to the time of appearance of symptoms and signs. Early onset complications typically appear hours to days post procedure while delayed onset complications usually develop weeks to years post HA filler injection. The most common side effects associated with HA injection are local injection related side effects which manifest as edema, pain, erythema, itching and ecchymosis. These adverse side effects are mild and usually last less than one week $[2,3]$.

In the 3rd case all the symptoms of edema- pain- erythema-itching were happened bur the ecchymosis was very mild. So the treatment with our protocol including quinolone antibiotic, solumedrol, Praxilen and laser He-ne seem be effective and the skin recovered very quickly. Ecchymosis and edema can be minimized by stopping the intake of aspirin, NSAID, supplements containing ginkgo biloba, vitamin E, omeg-3, fish oil, ginseng, kava- kava and St John's wort at least one week prior to the procedure. Before and after procedure use of arnica, topical vitamin $\mathrm{K}$ or bromalin may decrease the post-injection ecchymosis, but no controlled studies prove their effectiveness. Some practitioners use the vascular laser to reduce post-injection bruises [4].

- Pain is considered to be a common adverse effect during HA injection. Several techniques can be used in order to minimize the pain associated with injections, which include: the utilization of the small needle gauge or blunt-tipped cannulas, the use of topical anesthetic agents, application of ice prior and after injection, vibratory distraction and nerve blocks [5]. In all 3 patients they felt painful during the injection and afterward. 
- Tissue necrosis: We have 2/3 patients suffered from tissue necrosis in different category. The main high risk facial zones for skin necrosis and embolization are the glabella, nasal ala and dorsum of the nose. Several measures can be taken to minimize the risk of vascular complications including: through understanding of the facial anatomy, aspiration before each injection, low pressure injections of minimal volumes $\quad(<0.1$ $\mathrm{ml} /$ injection), dilution of the filler with lidocaine and/or epinephrine, keeping the needle moving (bolus injections should be given only in the periosteum plane), avoid injections in areas of previous scarring and use of blunt cannulas, which may reduce the risk of intravascular placement of the filler material Injectable fillers are also associated with infections, which can result from the breach in skin surface integrity[6].

- Antibiotics use: The infectious agents may be bacterial, viral or fungal. In order to minimize the risk of infection, the patients' history should be taken, including any history of recent dental procedures, any periodontal treatment planned within the next two weeks or any history of chronic sinusitis. The patient should not wear makeup either before or immediately after the procedure. Aseptic technique should be used, including proper skin sterilization with $2-4 \%$ chlorhexidine or $70 \%$ isopropyl alcohol solution and avoiding contamination of the treatment area after cleansing the patient's skin. An injection approach should be used that reduces the number of skin piercings and uses the smallest gauge needle possible for injections.

Bacterial inoculations can occur after filler injections as a result of skin surface breakage. Inflammatory nodules that present with erythema, edema and tenderness, in other words, a "red angry bump", which presents within 3-14 days should be treated as an infection. Another approach is to aspirate the lesion with an 18-gauge needle after applying topical anesthesia. The patient should be started on empiric broad-spectrum antibiotics immediately, selecting drugs that provide coverage against acid-fast bacilli, atypical mycobacteria [7].

- Injection of Hyaluronidase: We have 2/3 patients had Hyaluronidase injected after the complication happened. Intralesional hyaluronidase is an effective therapy for granulomatous lesions secondary to HA filler. Hyaluronidase was injected into the granulomatous nodules with a complete resolution of the skin lesions. Other treatments that can be used to resolve granulomas include systematic and intralesional corticosteroids, systemic oral antibiotics, intralesional5-fluorouracil and laser treatment

- Visions loss: Blindness is the most feared complication of fillers injection. It has been proposed that accidental high injection pressure of the supratrochlear, supraorbital, angular and dorsal nasal arteries which are branches of the external carotid artery will result in a retrograde flow of the filler emboli into the ophthalmic artery. Once the physician stops the pressure on the plunger, the arterial pressure will push the filler emboli into the retinal circulation resulting in the loss of vision. If the physician applies a greater force for a long time, the filler emboli can reach the internal carotid artery and then be propelled into the intracranial circulation resulting in cerebral ischemic events. Luckily, our patients were not suffered from blindness [8]. 


\section{CONCLUSION}

HA fillers injection for aesthetic purposes are the predominantly used dermal fillers worldwide. However, the side effects of HA injection is a big and serious problem in Vietnam now. Although most complications associated with HA filler injection are mild and self-limiting but the vascular and infectious complications associated with HA filler injection can be terrible consequences for patients. We can minimize these complications with a thorough understanding of facial vascular anatomy, proper injection techniques and meticulous skin preparation. Early identification and a prompt intervention can significantly decrease the risk of long-term sequelae. Early recognition of vascular necrosis with specific protocol for treatment after injection necrosis with hyaluronic acid fillers improves the outcome of wound healing.

\section{REFERENCES}

1. Bailey, S.H., Cohen, J.L., Kenkel, J.M., 2011. Etiology, prevention, and treatment of dermal filler complications. Aesthet. Surg. J. 31 (1), 110- 121.
2. Beer, K., Downie, J., Beer, J., 2012. A treatment protocol for vascular occlusion from particulate soft tissue augmentation. J. Clin. Aesthet. Dermatol. 5 (5), 44-47.

3. Bray, D., Hopkins, C., Roberts, D.N., 2010. A review of dermal fillers in facial plastic surgery. Curr. Opin. Otolaryngol. Head Neck Surg. 18 (4), 295-302.

4. Brody, H.J., 2005. Use of hyaluronidase in the treatment of granuloma- tous hyaluronic acid reactions or unwanted hyaluronic acid misplace- ment. Dermatol. Surg. 31 (8 Pt 1), 893-897.

5. Cassuto, D., Marangoni, O., De Santis, G., Christensen, L., 2009. Advanced laser techniques for filler-induced complications. Dermatol. Surg. 35 (Suppl 2), 1689-1695.

6. Cohen, J.L., 2008. Understanding, avoiding, and managing dermal filler complications. Dermatol. Surg. 34 (Suppl 1), S92-S99.

7. Cohen, J.L., Biesman, B.S., Dayan, S.H., DeLorenzi, C., Lambros, V.S., Nestor, M.S., et al., 2015. Treatment of hyaluronic acid filler-induced impending necrosis with hyaluronidase: consensus recommendations. Aesthet. Surg. J.

8. Jean Carruthers, A.C. (Ed.), 2013. Soft Tissue Augmentation, third ed. Elsevier Saunders. Complications of minimally invasive cosmetic procedures: prevention and management. J. Cutan. Aesthet. Surg. 5 (2), 121-132. 